\title{
Review Article Delay Tolerance in Underwater Wireless Communications: A Routing Perspective
}

\author{
Safdar Hussain Bouk, Syed Hassan Ahmed, and Dongkyun Kim \\ School of Computer Science and Engineering, Kyungpook National University, Daegu, Republic of Korea \\ Correspondence should be addressed to Dongkyun Kim; dongkyun@knu.ac.kr
}

Received 24 September 2016; Accepted 21 November 2016

Academic Editor: Yujin Lim

Copyright (C) 2016 Safdar Hussain Bouk et al. This is an open access article distributed under the Creative Commons Attribution License, which permits unrestricted use, distribution, and reproduction in any medium, provided the original work is properly cited.

\begin{abstract}
Similar to terrestrial networks, underwater wireless networks (UWNs) also aid several critical tasks including coastal surveillance, underwater pollution detection, and other maritime applications. Currently, once underwater sensor nodes are deployed at different levels of the sea, it is nearly impossible or very expensive to reconfigure the hardware, for example, battery. Taking this issue into account, considerable amount of research has been carried out to ensure minimum energy costs and reliable communication between underwater nodes and base stations. As a result, several different network protocols were proposed for UWN, including MAC, PHY, transport, and routing. Recently, a new paradigm was introduced claiming that the intermittent nature of acoustic channel and signal resulted in designing delay tolerant routing schemes for the UWN, known as an underwater delay tolerant network. In this paper, we provide a comprehensive survey of underwater routing protocols with emphasis on the limitations, challenges, and future open issues in the context of delay tolerant network routing.
\end{abstract}

\section{Introduction}

Communication and monitoring of underwater environment have been long-standing challenges in a vast range of applications that are enabled by underwater wireless communications. The scope of these applications ranges from monitoring ocean pollution to environment, climate, natural disturbances, and marine ecosystem for search and survey operations, surveillance, offshore exploration, navigation, and disaster prevention. Applications such as the above mentioned have led to a flurry of research investigating how better communication and monitoring could be achieved in underwater wireless communications [1].

Networks that enable underwater communication are called underwater wireless networks, underwater acoustic networks, or underwater acoustic sensor networks, as shown in Figure 1. However, in this context, we refer to them collectively as underwater wireless networks (UWNs). A UWN is composed of offshore and onshore static and mobile nodes. The offshore static nodes are battery operated, with sensing capability, anchored to or resting on the seabed, and floating at different heights. The other set of nodes floating over the sea surface or under the sea, either autonomously or manually controlled, are called offshore mobile nodes [2].

The main objective of UWNs is to sense and monitor underwater environment as well as control autonomous underwater vehicles (AUVs) and provide this information to an offshore center through intermediate data collection points or sinks floating over the sea surface [3]. Each UWN node below the sea surface is equipped with sensing functionality to collect features and parameters of the underwater environment. This sensed data is forwarded to the onshore stations through stations floating over the sea surface (e.g., boats or buoys) in a multihop fashion [4].

Diverse communication modes, for instance, radio frequency (RF) electromagnetic waves [5] and acoustic and/or optical signals [6], are used between offshore surface stations and onshore data centers as well as among offshore nodes. $\mathrm{RF}$ electromagnetic waves are used to enable communication between nodes that are floating over the sea surface and onshore data collection and control center. However, the same cannot be applied to propagate information between the wireless nodes below the sea surface because sea water is not a good conductor of electromagnetic waves due to 

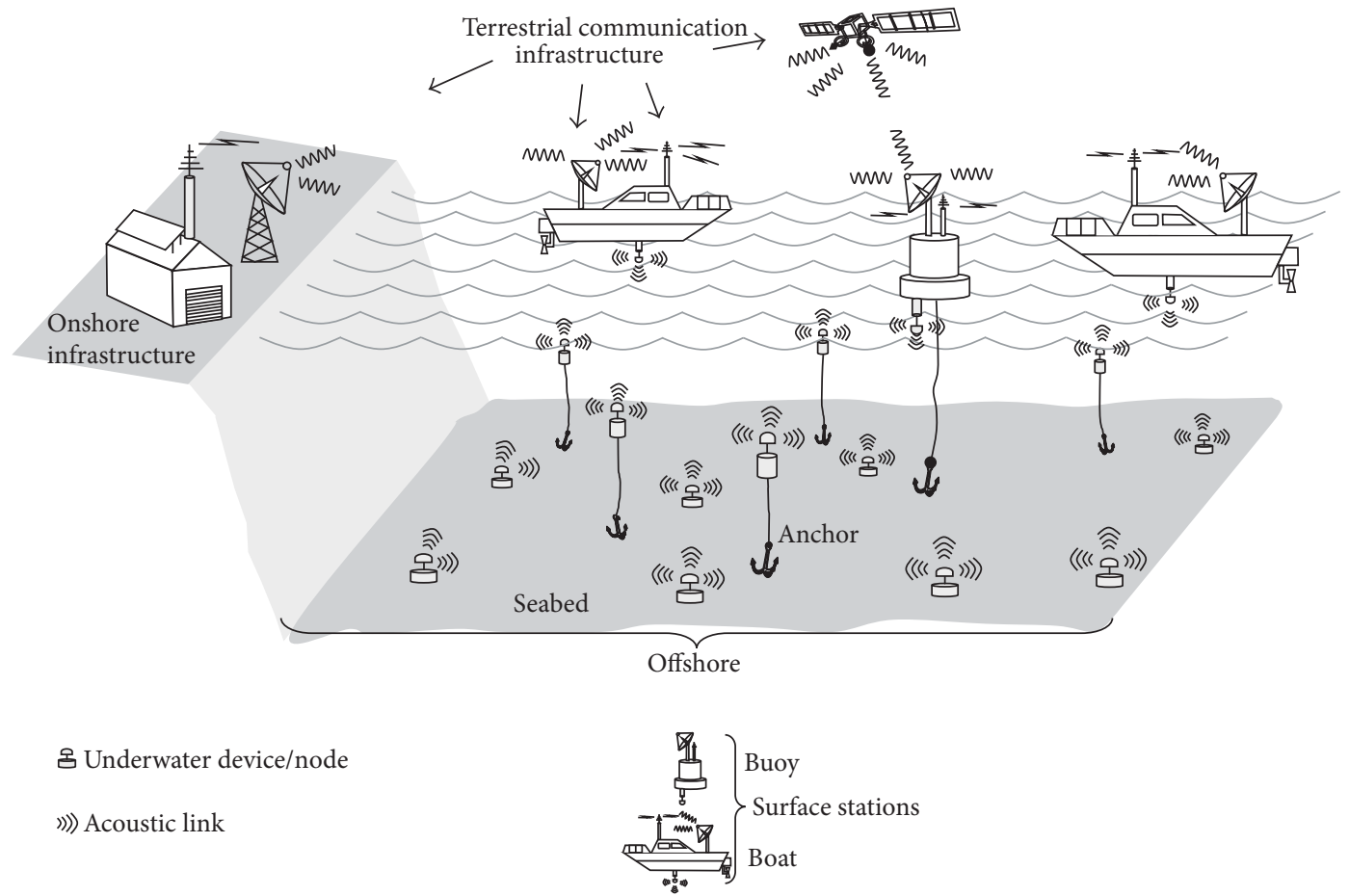

FIGURE 1: Underwater wireless communications network.

salinity. The alternative communication mode in underwater environment is optical communication. However, in a turbid underwater environment, optical communication severely suffers from high scattering, which makes it impracticable for underwater communication.

Other than the RF electromagnetic and optical signals, acoustic signals are employed as an enabling communication mode in the UWNs. Acoustic signals can propagate efficiently in the aquatic environment for very long distances (measured in kilometers). However, acoustic signals and aquatic channels collectively put forward many constraints and challenges that need to be overcome. These constraints pose an uncertain end-to-end path between source and destination nodes as well as frequent network partitions and intermittent links between nodes. This makes real-time communication a challenging task in UWNs [7]. Hence, it is necessary to get aware of the current state-of-theart routing solutions and understand the limitations of an acoustic channels that hinder the data forwarding in such a constrained environment. In this article, we therefore provide a comprehensive overview of routing in underwater communications. The key contributions of this article are as follows:

(i) An overview of the limitations of acoustic signals and channels (Section 2)

(ii) A brief discussion on the traditional routing classes for UWN (Section 3)

(iii) An introduction to the underwater DTN and state-ofthe-art routing solutions (Section 4) (iv) Detailed discussion and unique classification and comparison of underwater DTN routing protocols (Section 5)

(v) Enlisted shortcomings of existing solutions along with the future research directions (Section 6)

\section{UWN Limitations}

The comprehensive list of acoustic signals and aquatic channel limitations of the UWN [8] is as follows.

(1) Propagation Delay. The main constraint of acoustic signals in underwater is its high propagation delay that is approximately $2 \times 10^{5}$ times slower than the electromagnetic propagation in terrestrial environments. The acoustic delay is highly dynamic and contingent on different factors that include water temperature, depth, and salinity.

(2) Multivariate Attenuation. The acoustic signal faces severe path loss that is inherent in the acoustic channel and mainly depends on signal frequency, absorption loss, sender-receiver distance, and pathloss exponent.

(3) Effects of Noise. The frequency band of the acoustic channel is constrained by the effect of ambient noise in the aquatic region. There are three main categories of ambient noise sources: (i) marine animals, including Dolphins, whales, and snapping shrimp., (ii) human activity that consists of on- and offshore exploration, industrial activity, ships, fishing, and 
communication, and (iii) natural events, ranging from underwater earthquakes to ice cracking, volcano eruption, bubbles, rain, lightning, and wind to the surface waves.

(4) Limited Bandwidth. The acoustic bandwidth is reduced to a small range by varying factors that include high impact of sound at lower frequencies and large medium absorption at higher acoustic frequencies.

(5) Time-Varying Multipath Propagation. Each acoustic ray generated by an acoustic source follows a different path with varying delays. Multiple paths are followed by each ray due to boundary reflections (e.g., sea surface, seabed) or other objects suspended in the sea water, and refraction is caused by varying sound profiles at different depths. In response, the distant receiver will receive multiple copies with varying strengths and delays that result in unstable phase response.

(6) High Transmission Power. High transmission power is required to propagate the acoustic signal, which constrains the network lifetime.

(7) Bit Error Rate. High bit error rate occurs as a result of time-varying characteristics and multipath interference characteristics of the acoustic channel.

(8) Intermittent Connectivity. Temporary loss of connectivity is experienced due to shadow zones (the regions where acoustic signal does not reach or intensity of the signal is very low).

(9) Limited Energy Harvesting Options. Energy harvesting is difficult in the underwater environment, which restricts the UWN nodes to limit their wireless communication duration to prolong the network lifetime.

(10) No Position Information. Unpredictable ocean currents and intrinsic characteristics of the aquatic channel make it difficult to provide position information in UWNs.

The aforementioned constraints fail to provide deterministic end-to-end connectivity between source and destination and successive network partitions. With all these limitations, it is hard to achieve real-time communication. Therefore, the existing routing solutions for terrestrial networks cannot be directly applied to the UWNs. Therefore, a new paradigm of routing protocols that tolerates the delays and disruptions, called the delay tolerant network (DTN) routing in the UWN, has been explored by researchers.

Fall [9] introduced the concept of a Delay Tolerant Networks by proposing the architecture. In the DTN architecture, the authors characterized communication delay and frequent network partitioning due to the intermittent connectivity. Designing routing protocols for such networks is always a challenge as there is no continuous path from source to destination and hence conventional routing protocols usually fail. To the best of our knowledge, every DTN routing protocol follows a store-carry and forward mechanism, where sensor nodes need to keep messages in buffer until they meet an intermediate node or destination directly (most of schemes do not consider direct delivery enabling relay based routing in DTN). A representative scheme for DTN storecarry and forward (SCAF) approach is Epidemic routing [10], which forwards each copy of message to each node. Epidemic routing scheme is flooding based in nature, thus exhausting network resources. In order to deal with a resource utilization issue, Wang et al. proposed Spray and Wait [11], in which only a limited number of message copies are replicated among nodes. One improvement in DTN for vehicles is MaxProp [12], where priority is given to nodes on some criterion and also messages are also characterized while forwarding.

Data forwarding in underwater DTN is being conducted with the SCAF mechanism. In case of unavailability of nexthop connectivity, an intermediate node stores the received packet(s). The packets are forwarded to the next-hop node when the opportunistic connectivity to the next-hop node is available. This type of approach provides eventual data delivery to the destination with a certain probability. The authors in [13] provide a survey of the generalized routing protocols for the underwater DTNs and classify them based on the nature of the link contact between the UWN nodes.

\section{Routing Features in UWNs}

Reliable and energy efficient data delivery with minimal delay from source to destination nodes in battery-operated multihop networks is prime design goals of the routing layer. An efficient routing protocol first discovers suitable path(s) and then forwards data towards the destination node by following the selective path(s) [14]. There is a plethora of routing solutions proposed for terrestrial multihop networks. Similar routing solutions have also been tested in the UWNs and have been shown to cause poor performance due to intrinsic characteristics of the UWNs.

In terrestrial multihop networks, the routing protocols are classified on the basis of network characteristics, route discovery, routing information management, and/or data communication mechanism. The classification parameters include path discovery mechanism (on-demand and periodic or reactive and proactive), network topology (flat and hierarchical), topology management (centralized and distributed), number of paths (single and multiple paths), data communication mechanism (unicast, multicast and broadcast), and location information (geocasting with or without flooding). Similarly, the classification of UWN routing protocols revolves around identical features, as shown in Figure 2, some of which are discussed below.

Node Position/Localization. Location information such as the global positioning system (GPS) is not directly available to the UWN nodes under the sea surface. However, the nodes floating at the sea surface receive location information and share it with the underwater nodes through acoustic beams. Similarly, the position information of the nodes below the sea surface is acquired through depth and pressure sensors. Routing protocols that perform data forwarding based on the above information are classified as location-based, depthbased, and pressure-based routing algorithms. 


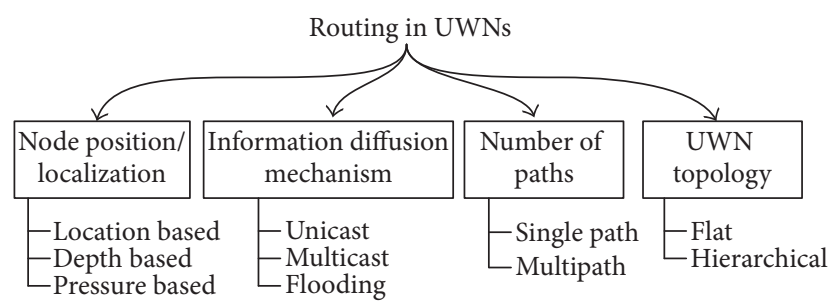

FIGURE 2: General classification of UWN routing protocols.

Information Diffusion Mechanism. Efficient information diffusion is the prime objective of any routing protocol. The UWN routing protocols use various information propagation methods and are classified accordingly. For example, information may be disseminated to single, multiple, or all the UWN nodes in unicast, multicast, or broadcast manner, respectively. However, each method has its own drawbacks. For example, high control overhead is required to maintain the UWN topology information in unicast, while broadcast or flooding consumes large amount of energy because of message duplication in the network.

Number of Paths. Data from the UWN source node(s) towards the destination or sink node are forwarded through single or multiple paths. The multiple path formation algorithms enhance reliability and robustness in data delivery in an intermittent communication environment such as the UWN.

UWN Topology. The UWN routing protocols are further classified in terms of network topology as flat or hierarchical. In a flat topology UWN, all nodes equally participate in the routing task, and the routing protocol has high control overhead to maintain topology information. In contrast, in hierarchical topology UWN, the routing protocols divide the network into small groups, called clusters, based on localization or other parameters. Each cluster is assigned a group head or cluster head that efficiently manages the network resources among the group members. As a result, spatial reuse of network resources can be achieved within the network, which alleviates network capacity.

Mobility Influence. To sense a vast underwater area, the underwater networks are sparsely deployed due to the high cost of underwater sensor nodes, which results in a delay or disruption tolerant underwater network. The sparsity may also have resulted due to oceanic and natural forces, for example, water currents, the wind, and water depth [15]. Therefore, the AUVs are used to collect data from the sparse network. Mostly, the AUVs have a planned network traversal path that is suitable for the static or quasi-static networks, to collect data from the network. This path may be computed based on the sufficient contact duration between the nodes to collect the data. However, the underwater sensor node mobility may disrupt this contact duration that will change the network performance. In addition to that, various underwater communication technologies have diverse characteristics of transmission range, bandwidth, communication delay, and power consumption. For example, an acoustic channel has long range, small bandwidth, and high delay and radio frequency plus Magnetic induction has short range, high bandwidth, and moderate delay. Therefore, it is difficult to choose how fast or how far the communication is required. Thus, the mobility pattern of AUVs and the deployed underwater nodes have a significant impact on the performance of the routing schemes in the underwater DTN.

\section{Routing in Underwater DTN}

The concept of DTN refers to the network architecture that exhibits communication delay and frequent network partitioning due to intermittent connectivity. Designing routing protocols for such networks is always a challenge as there is no continuous path from source to destination and, hence, conventional routing protocols usually fail. Therefore, the recent paradigm shift drives us towards such routing solutions that ensure network performance at lower network resource costs.

Every underwater DTN routing protocol follows a SCAF mechanism, where underwater nodes need to store messages in their buffer until they encounter an intermediate node or destination directly, as shown in Figure 3. Therefore, each underwater DTN routing protocol must address the above constraints of DTN and provide energy efficiency, minimum end-to-end delay, and maximize overall network performance.

The authors in [13] survey the existing underwater DTN routing protocols and classify them based on the nature of the communication link between nodes. There are two main sets of routing protocols that assume that scheduled communication links or contact between nodes can be scheduled or unscheduled. Furthermore, the unscheduled contact schemes are subdivided into opportunistic and predicted contact. Please refer to [13] for more details.

In this survey, we provide an overview of recent advancements in underwater DTN routing schemes and classify them based on the forwarding and routing decisions, copies of messages, and performance metrics. Following is the detailed description of the classification parameters.

Communication Initiation Mechanism. Generally, data communication achieved by underwater network protocols is either Sender-initiated (push-based) or Receiver-initiated (pull-based). In the former, the data sender node selects its next-hop sensor node that relays the data further to the indicated destination. However, in the latter, the receiver node initiates the request to start the data communication.

Infrastructure Assistance. The underwater DTN routing schemes that perform routing decisions based on the information provided by the infrastructure nodes, for example, gateway(s), node(s) mounted on buoy(s), boats, and ships, are called infrastructure-assisted schemes. These infrastructure nodes periodically or on-demand maintain the network topology information and this information is made available to each node in the underwater DTN. 


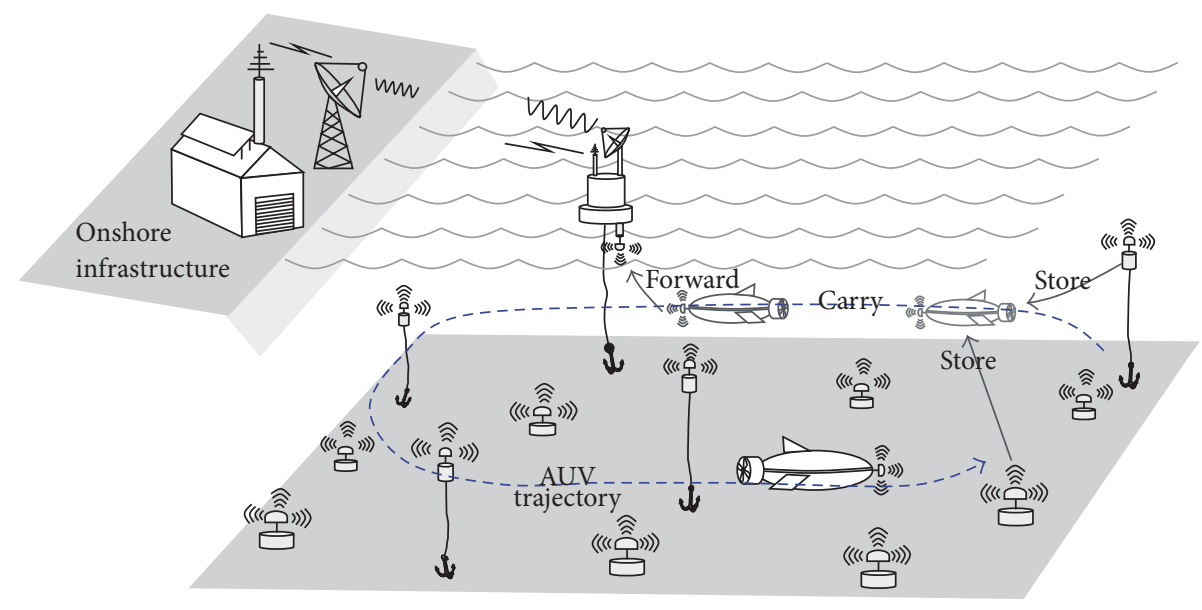

FIGURE 3: Store-carry-and-forward mechanism in UWNs.

Routing Decision. Routing decision is defined as the method by which an underwater DTN node selects next-hop based on local or global network topology information. We classify routing decisions into two main categories, namely, centralized or distributed. The routing protocols that perform routing decisions based on the global topology information are called centralized schemes. On the other hand, the underwater DTN nodes perform forwarding decisions subject to the locally discovered topology information. These schemes are termed as distributed routing schemes.

Location Information Dependence. The location information is one of the main routing metrics to carry out efficient routing. We consider the location information as one of the metrics to categorize the underwater DTN routing schemes.

Number of Message Copies. The source nodes in underwater DTN routing protocols generate and forward single or multiple copies of the same data messages in the network to ensure data delivery to the destination node.

Performance Indicator. Each routing protocol is proposed with a motive to improve network performance indicator metric(s). That performance metric can be throughput, endto-end delay, control overhead, energy consumption, network lifetime, and so on.

Next, we briefly survey the state-of-the-art underwater DTN routing schemes by maintaining the above classification parameters, as shown in Figure 4, as a baseline for comparison.

\section{Routing Protocols for Underwater Delay Tolerant Networks}

Recently, many routing protocols for underwater DTN have been proposed to improve network performance under the adverse underwater environment. Following is a brief survey of recent progress in terms of research on routing protocols, especially for underwater DTNs.

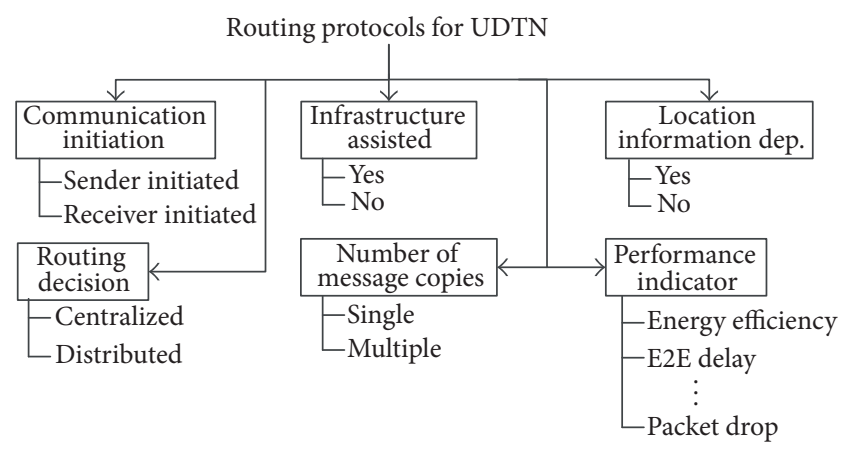

FIgURE 4: Classification parameters of underwater DTN routing protocols.

5.1. PASR. Guo et al. [16] proposed a generic scheme called prediction-assisted single-copy routing (PASR) for underwater DTNs to minimize delivery delay. PASR is a singlecopy routing protocol where the authors divided routing operations into two phases, namely, learning and routing phases. In the learning phase, short duration traces are collected while connecting the network and characterization is performed by using an offline greedy algorithm. An aggressive chronological projected graph (ACPG) algorithm is used for the learning phase. As we know, if a mobility pattern of nodes is stable for a maximum time, its history can be predicted. In the routing phase, historical information such as a recent trajectory, duration of average contact and intercontact, and last contact and frequency of contacts is collected and used to predict mobility routes. On the basis of those patterns, a sender adaptively selects the most optimal routes while forwarding the data to its next hop. The authors in [17] proposed an adaptive routing scheme for underwater delay/disruption tolerant networks. The scheme assumes that each node knows its $3 \mathrm{D}$ position through localization schemes. The priority of any data packet is calculated and assigned on the basis of varying properties of a node and data packet including node density, residual energy, age of packet, 
and urgency of packet. The protocol achieves a good tradeoff between energy consumption, delay, and delivery ration.

5.2. DDD. E. Magistretti et al. [18] proposed a delay tolerant data dolphin (DDD) approach to apply in a resourceconstrained underwater environment. For ensuring energy efficiency, mobile collectors, called Dolphins, are introduced. In order to limit the utilization of multihop channels, the underwater sensors are obligated to communicate within a single hop when a Dolphin is within transmission range. The rationale is to increase the overall network lifetime. Hence, the sensors can save power by limiting their transmission to only mobile Dolphins and avoiding multihop communication. Once data are delivered, the sensors' memory is flushed out and data are secured in the Dolphins' memory. Dolphins are prepared to transmit periodic beacons to inform the sensors about their arrival in the region. As soon as nodes determine the presence of a Dolphin, they turn on acoustic modems and upload stored events to the Dolphin. Later, when the Dolphin moves within the communication range of a base station, it uploads all gathered events. However, adjusting the optimal speed of the Dolphin and its trajectory is still an important issue.

5.3. Azad et al. UDTN. Autonomous coastal surveillance is one of several applications for underwater networks. Mostly, AUVs are deployed under the shores to monitor a given area. However, AUVs depend on a fixed trajectory. Even if the trajectory is adaptive, in case of critical data, they require time to reach a base station for data delivery. For coping with the issue, Azad et al. [19] proposed a potential communication between two AUVs that can minimize the delay in data delivery during coastal surveillance. Generally, the given area is quite large, so multiple AUVs are deployed and each AUV aims to reach the base station after completing its full-length trajectory, which causes a very long delay. Hence, the core idea is to forward the data between two or more AUVs after exchanging beacon, information, and response messages. The information message is sent as a response to the beacon and it includes the identities, routes, and movement speed of AUV(s). This information ensures the decision-making, that is, either transmitting data to the $\mathrm{AUV}(\mathrm{s})$ passing by or not.

5.4. Sweeper. One of the important factors affecting wireless communication is antenna capabilities. In [12], the authors proposed a scheme called Sweeper that considers a central node with an adaptive directional antenna array. This antenna array forms a beam in a specific direction and width, called downlink, which implies that the central node can communicate with the normal underwater sensor nodes within the specific region. The central node sends a wakeup or query message, which consists of a specific task for the normal nodes in the specific region through downlink. The receiving node(s) first calculate(s) depth level using a received signal strength indicator to estimate the region. After successful reception of the wakeup message, the normal nodes in the region send their sensed data towards the central node through the same direction of arrival, that is, uplink direction. In this manner, the central node sweeps the area to collect data and keeps track of location information of the normal nodes in the region.

5.5. Tier-Based Cross-Layer. A tier-based cross-layer routing protocol for the underwater DTN has been proposed in [20]. The network is divided into tiers with a reference from the sink node. In one tier, both transmitter and receiver should be in each other's transmission range and the maximum range is defined as $d_{\max }$. The value of $d_{\max }$ is determined to achieve a certain level of channel capacity, because the channel capacity is highly affected by the distance between transmitter and receiver. The cross-layer optimization is achieved by synchronizing the MAC and routing functionalities together to minimize collision that causes the delay. If a node in tier $j$ wants to communicate with the sink, it sends its tier information in a request-to-send message and the node(s) in the upper tier, $j 1$, reply with clear-to-send (CTS) message. After receiving multiple CTSs from the upper tier nodes, the source node sends intent-to-send with the node number that acts as a next-hop routing node. The selection of the next-hop node is done based on the minimum distance that requires small transmit power. As a result, the proposed protocol has minimum delay and high accuracy rate and consumes less energy.

5.6. QDTR. In [10], authors proposed Q-Learning-based routing protocol for underwater DTNs. Q-Learning is one of the reinforcement learning algorithms that is used to take optimal data-forwarding decisions. In the given protocol, underwater nodes forward single or multicopy packets at the beginning and spray packets among the neighbors of the destination at the end, which achieves better network performance and energy.

However, the protocol does not assume any node mobility pattern, as normally used in terrestrial DTNs, where future meeting events can be predicted with the aid of the mobility model. The proposed protocol is able to adapt to packet priority. When the deadline is approaching, nodes forward more copies of packets than usual, which is a tradeoff between higher energy and shorter delay.

5.7. RBAR. Redundancy Based Adaptive Routing (RBAR) [21] for underwater DTNs has been proposed allowing a node to hold packet as long as possible until it has to make another copy to satisfy its delay requirement. In order to achieve this goal, RBAR takes advantage from a binary tree based forwarding procedure, which helps packet replication process to be explicitly determined. The main contribution of RBAR is packet-forwarding process.

5.8. PBDTP. Zhang et al. intended to address channel throughput issue for underwater DTNs [22]. Due to inconsistent delay in acoustic channel, many traditional communication protocols are insufficient since they depend on accurate estimations of RTT, that is, Round Trip Time between two nodes. In order to deal with long delays, a Prediction-Based Delay Tolerant Protocol has been proposed, where prediction for lost data by any sensor node with in cluster is performed by cluster head. The core idea behind this protocol is based 
TABLE 1: Summary of underwater DTN routing protocols.

\begin{tabular}{|c|c|c|c|c|c|c|}
\hline $\begin{array}{l}\text { Routing } \\
\text { protocol }\end{array}$ & $\begin{array}{c}\text { Communication } \\
\text { initiation } \\
\text { mechanism } \\
\end{array}$ & $\begin{array}{l}\text { Infrastructure } \\
\text { assistance }\end{array}$ & $\begin{array}{l}\text { Routing } \\
\text { decision }\end{array}$ & $\begin{array}{c}\text { Location } \\
\text { information } \\
\text { dependence }\end{array}$ & Number of copies & $\begin{array}{l}\text { Performance } \\
\text { indicator }\end{array}$ \\
\hline PASR & Sender & No & Distributed & No & Single & $\begin{array}{c}\text { Replication } \\
\text { control }\end{array}$ \\
\hline DDD & Receiver & No & Centralized & No & Single & Energy efficiency \\
\hline UDTN & Receiver & No & Distributed & No & Single & $\begin{array}{l}\text { End-to-end delay } \\
\text { minimization }\end{array}$ \\
\hline Sweeper & Receiver & Yes & Centralized & Yes & Single & $\begin{array}{l}\text { UW nodes } \\
\text { localization }\end{array}$ \\
\hline $\begin{array}{l}\text { Tier-based } \\
\text { UDTN }\end{array}$ & Sender & Yes & Centralized & Yes & Single & $\begin{array}{c}\text { Energy, delay, and } \\
\text { packet drop } \\
\text { constraints }\end{array}$ \\
\hline QDTR & Sender & No & Distributed & No & Multiple & $\begin{array}{c}\text { Energy efficiency } \\
\text { and end-to-end } \\
\text { delay }\end{array}$ \\
\hline RBAR & Sender & Yes & Centralized & No & Multiple & Energy efficiency \\
\hline PBDTP & Sender & Yes & Not Available & No & Multiple & $\begin{array}{l}\text { Long delay } \\
\text { tolerance }\end{array}$ \\
\hline
\end{tabular}

on two assumptions: (a) a sensor node most likely will send the same data with small time interval and (b) adjacent sensor nodes will also report almost identical sensed data. In case of lost data packet, the proposed algorithm predicts the missing value based on either previous values of specific sensor node or its neighbor's values. On the other hand, prediction is also being done by combination of both. In meanwhile, the lost data packets are compensated at cluster head nodes avoiding extra delays caused by sending retransmission requests and waiting for sensor to resend data.

Table 1 shows the summary of underwater DTN protocols in terms of the previously discussed classification parameters. It shows that PASR utilizes the offline greedy algorithm for mobility prediction. Based on mobility patterns archived in the learning phase, two different schemes have been tested for random mobility traces [16]. While selecting a next-hop, a sender has information about the most optimal route for a destination. This leads to low congestion and an overhead transmitting single copy of the data. Therefore, it belongs to the sender-initialized communication.

Controversially, DDD [18] uses Dolphins, mobile AUVs, as data collectors. Dolphins initiate communication and collect data from the one-hop UWN nodes while on the move. On successful reception of data by the Dolphin, each UWN node refreshes its buffer. The tier-based underwater DTN routing scheme [20] is completely based on the infrastructure nodes. We argue that the infrastructure-dependent schemes have to rely on relevant ideal assumptions for showing better performance as compared to those with no dependency on infrastructure.

As mentioned before, the anatomy of overall network lifetime in UWN is very important. For achieving this, Sweeper [12] provides a centralized localization scheme where a central node sweeps the entire network to estimate the position of the sensor nodes. Further contrast between the selected protocols is elaborated in Table 1 . Next, we present the current challenges and open issues in underwater DTN routing.

\section{Challenges and Open Issues}

Here, we further identify the remaining challenges and issues, providing a concise guide for researchers. As we discussed earlier, routing is a key challenge in underwater DTNs where random mobility and intermittent environment complicate the acoustic channels. It is important to select the next hop using time-varying information while considering the usage of link quality and buffer space as well as energy. However, there still exist the following challenges and open issues that need to be investigated.

(i) The protocols using a multiple paths transmission strategy provide reliability and robustness. These protocols also have low end-to-end delays. However, robustness is further improved with the cost of retransmission. As repetitions limit the overall performance of a protocol and the complexity of maintaining more paths enlarges the processing overhead, efficient efforts are required to avoid retransmissions. More specifically, sending the same packet through multiple routes can be addressed in an intelligent manner.

(ii) There always exists a tradeoff between delivery ratio, energy constraints, and end-to-end delay while applying different approaches in the underwater DTNs. Artificial intelligence-aware routing can be expected to minimize the tradeoff.

(iii) Numerous routing protocols are based on historical information being used to select next-hop for communications. The problem is to determine the mechanism of information collection. The accuracy 
in case of random movements or future mobility of nodes can make it more challenging.

(iv) In most cases, researchers are emphasizing energy constraints while investigating routing issues. However, reallocating buffer space and maximum use of other resources can also be addressed in order to test algorithms in real-time experiments.

(v) In addition, a smart router should be configured to support many routing algorithms at once using a fuzzy logic technique. It should be a great effort to enhance and provide multiple routing decisions in a specific network.

Although many routing algorithms exist in the literature for DTNs and underwater acoustic networks, the number providing real-time analysis is still very limited. Therefore, there is a need for developing algorithms that facilitate realtime analysis.

\section{Conclusion}

In this article, we first provided an overview of routing in underwater communication and then presented delay tolerance features being adapted in the UWN research paradigm. The main goal of this survey is to make researchers and members of the industry familiar with the recent technology convergence in the field of underwater sensor networks. For that purpose, we summarized state-of-the-art routing protocols considering no end-to-end connectivity. In addition, we also provided a list of open challenges and future directions followed by a comparative analysis of the selected underwater DTN protocols. We hope this article will further motivate research interest in underwater DTNs.

\section{Competing Interests}

There are no competing interests regarding the publication of this paper.

\section{Acknowledgments}

This research was supported by the Basic Science Research Program through the National Research Foundation of Korea (NRF) funded by the Ministry of Education, Science and Technology (NRF-2016R1D1A3B01015510).

\section{References}

[1] A. Darehshoorzadeh and A. Boukerche, "Underwater sensor networks: a new challenge for opportunistic routing protocols," IEEE Communications Magazine, vol. 53, no. 11, pp. 98-107, 2015.

[2] D. Pompili and I. F. Akyildiz, "Overview of networking protocols for underwater wireless communications," IEEE Communications Magazine, vol. 47, no. 1, pp. 97-102, 2009.

[3] B. Li, Y. Xu, and W. Xu, "Multi-AUV localization for an underwater acoustic sensor network," in Underwater Acoustics and Ocean Dynamics, pp. 117-127, Springer, Singapore, 2016.

[4] J. J. Kartha, A. Jabbar, A. Baburaj, and L. Jacob, "Maximum lifetime routing in underwater sensor networks using mobile sink for delaytolerant applications," in Proceedings of the IEEE Region 10 Conference (TENCON '15), pp. 1-6, Macao, China, November 2015.

[5] D. Park, K. Kwak, W. K. Chung, and J. Kim, "Development of underwater short-range sensor using electromagnetic wave attenuation," IEEE Journal of Oceanic Engineering, vol. 41, no. 2, pp. 318-325, 2016.

[6] A. Caiti, E. Ciaramella, G. Conte et al., "OptoCOMM: introducing a new optical underwater wireless communication modem," in Proceedings of the IEEE 3rd Underwater Communications and Networking Conference (UComms '16), pp. 1-5, Lerici, Italy, August 2016.

[7] R. Grasso, P. Braca, S. Fortunati, F. Gini, and M. S. Greco, "Dynamic underwater sensor network for sparse field estimation," in Proceedings of the 3rd IEEE International Workshop on Compressed Sensing Theory and Its Applications to Radar, Sonar and Remote Sensing (CoSeRa '15), pp. 169-173, Genova, Italy, May 2015.

[8] M. Stojanovic and J. Preisig, "Underwater acoustic communication channels: propagation models and statistical characterization," IEEE Communications Magazine, vol. 47, no. 1, pp. 84-89, 2009.

[9] K. Fall, "A delay-tolerant network architecture for challenged internet," in Proceedings of the 2003 Conference on Applications, Technologies, Architectures, and Protocols for Computer Communications (SIGCOMM '03), pp. 27-34, ACM, Karlsruhe, Germany, 2003.

[10] T. Hu and Y. Fei, "An adaptive and energy-efficient routing protocol based on machine learning for underwater delay tolerant networks," in Proceedings of the 18th Annual IEEE/ACM International Symposium on Modeling, Analysis and Simulation of Computer and Telecommunication Systems (MASCOTS '10), pp. 381-384, August 2010.

[11] G. Wang, B. Wang, and Y. Gao, "Dynamic spray and wait routing algorithm with quality of node in delay tolerant network," in Proceedings of the IEEE International Conference on Communications and Mobile Computing (CMC '10), vol. 3, pp. 452-456, April 2010.

[12] K. T. Dharan, C. Srimathi, and S.-H. Park, "A sweeper scheme for localization and mobility prediction in underwater acoustic sensor networks," in Proceedings of the OCEANS'10 IEEE Sydney (OCEANSSYD '10), Sydney, Australia, May 2010.

[13] H.-H. Cho, C.-Y. Chen, T. K. Shih, and H.-C. Chao, "Survey on underwater delay/disruption tolerant wireless sensor network routing," IET Wireless Sensor Systems, vol. 4, no. 3, pp. 112-121, 2014.

[14] S. H. Ahmed, A. Wahid, and D. Kim, "EENC - Energy efficient nested clustering in UASN," in Proceedings of the 29th Annual ACM Symposium on Applied Computing (SAC '14), pp. 706-710, March 2014.

[15] A. Caruso, F. Paparella, L. F. M. Vieira, M. Erol, and M. Gerla, "The meandering current mobility model and its impact on underwater mobile sensor networks," in Proceedings of the 27th IEEE Communications Society Conference on Computer Communications (INFOCOM '08), pp. 771-779, Phoenix, Ariz, USA, April 2008.

[16] Z. Guo, B. Wang, and J.-H. Cui, "Generic prediction assisted single-copy routing in underwater delay tolerant sensor networks," Ad Hoc Networks, vol. 11, no. 3, pp. 1136-1149, 2013.

[17] Z. Guo, G. Colombi, B. Wang, J.-H. Cui, D. Maggiorini, and G. P. Rossi, "Adaptive routing in underwater delay/disruption 
tolerant sensor networks," in Proceedings of the 5th Annual Conference on Wireless on Demand Network Systems and Services (WONS '08), pp. 31-39, Garmisch-Partenkirchen, Germany, 2008.

[18] E. Magistretti, J. Kong, U. Lee, M. Gerla, P. Bellavista, and A. Corradi, "A mobile delay-tolerant approach to longterm energyefficient underwater sensor networking," in Proceedings of the Wireless Communications and Networking Conference (WCNC '07), pp. 2866-2871, March 2007.

[19] S. Azad, P. Casari, and M. Zorzi, "Coastal patrol and surveillance networks using AUVs and delay-tolerant networking," in Proceedings of the IEEE OCEANS, pp. 1-8, Yeosu, South Korea, May 2012.

[20] L. Kuo and T. Melodia, "Tier-based underwater acoustic routing for applications with reliability and delay constraints," in Proceedings of the IEEE International Workshop on Wireless Mesh and Ad Hoc Networks (WiMAN '11), pp. 1-6, Maui, Hawaii, USA, 2011.

[21] Z. Guo, Z. Peng, B. Wang, J.-H. Cui, and J. Wu, "Adaptive routing in underwater delay tolerant sensor networks," in Proceedings of the 6th International ICST Conference on Communications and Networking in China (CHINACOM '11), pp. 1044-1051, IEEE, Harbin, China, August 2011.

[22] Z. Zhang, S.-L. Lin, and K.-T. Sung, "A prediction-based delaytolerant protocol for underwater wireless sensor networks," in Proceedings of the International Conference on Wireless Communications and Signal Processing (WCSP '10), pp. 1-6, IEEE, Suzhou, China, October 2010. 

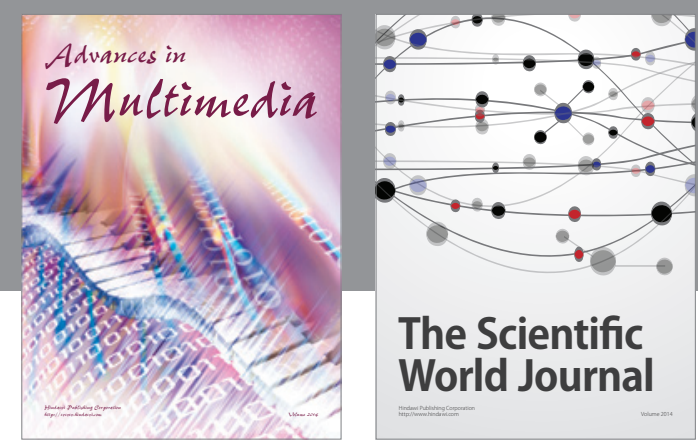

The Scientific World Journal
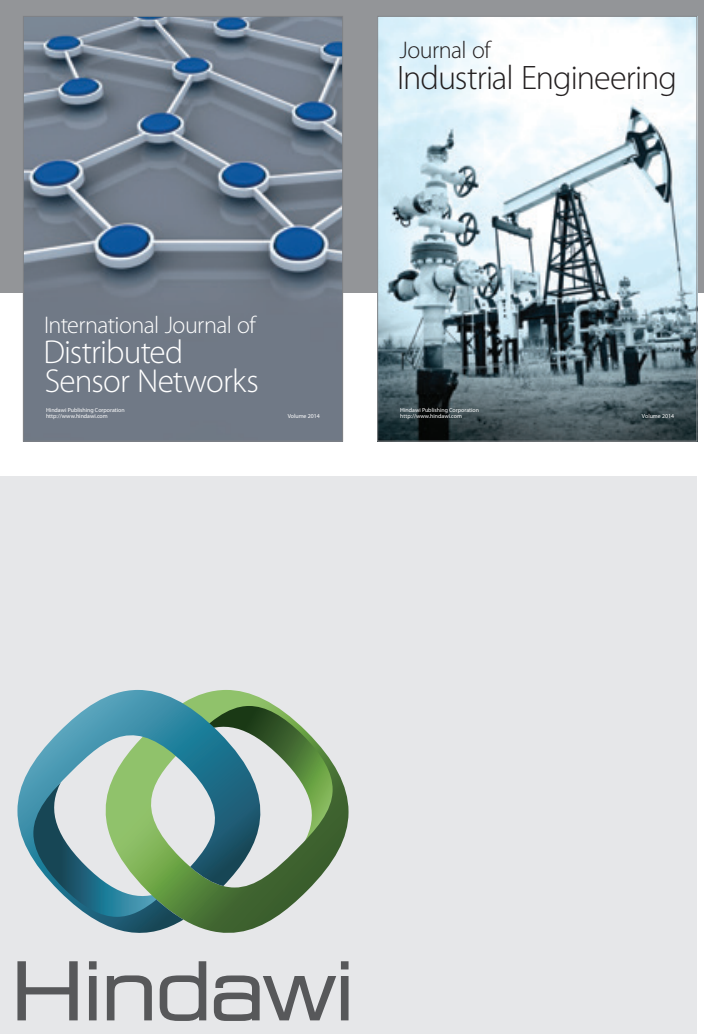

Submit your manuscripts at

http://www.hindawi.com

\section{Computer Networks} and Communications
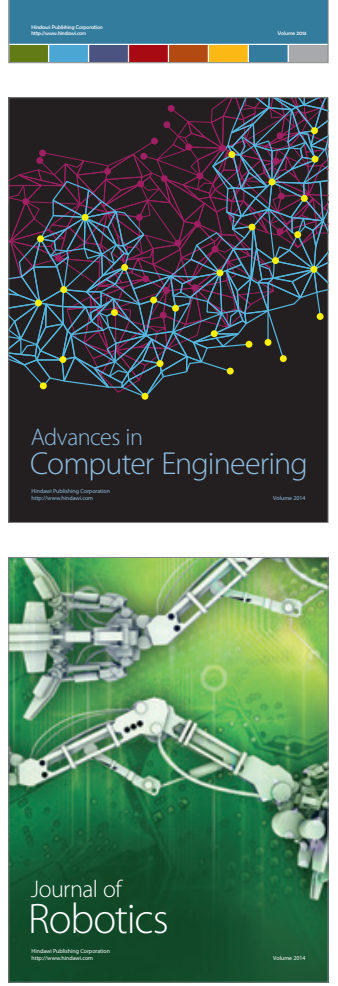
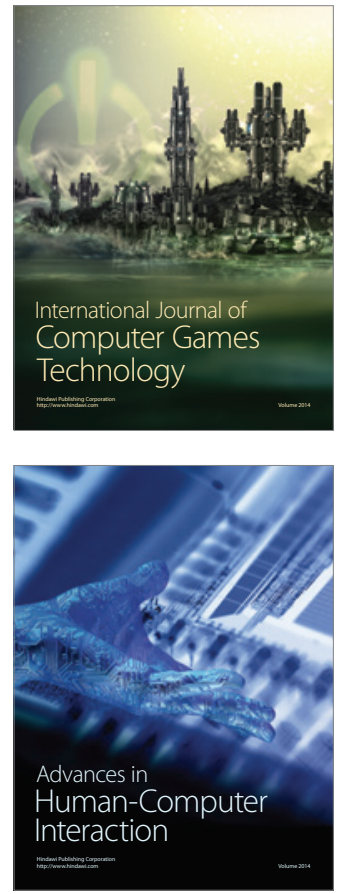
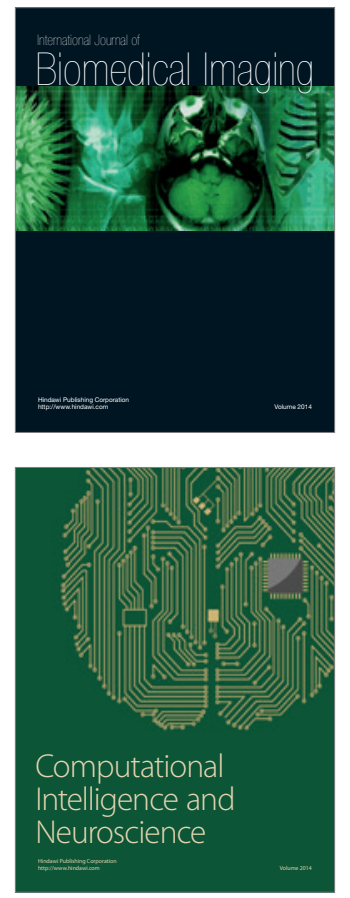
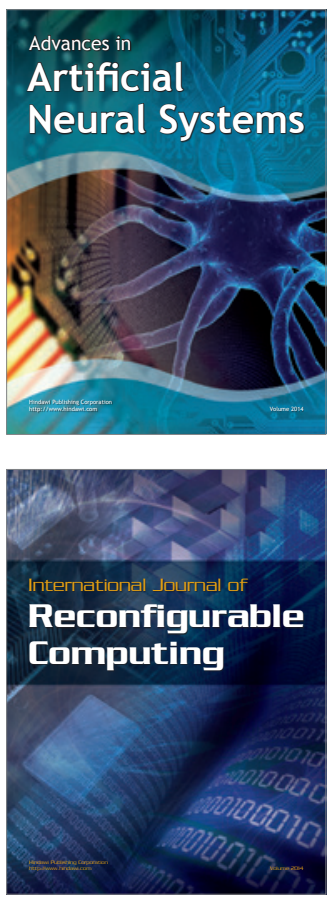
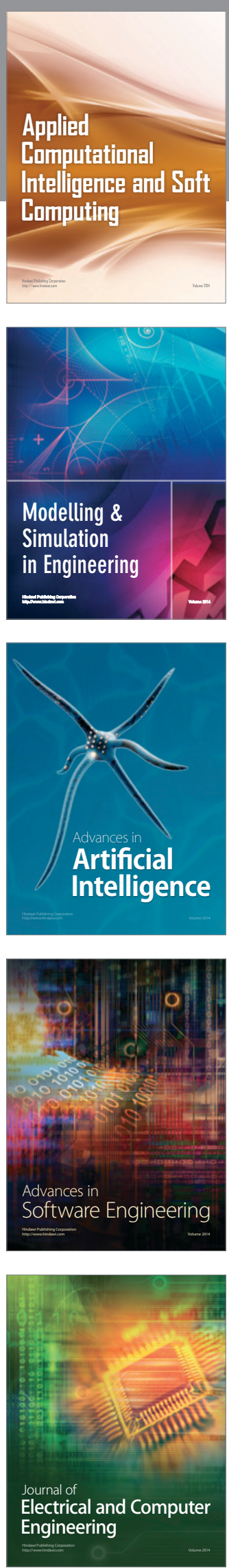